\title{
Surface Ionization of Radioactive Nuclides - Numerical Simulations
}

\author{
M. TUREK* \\ Institute of Physics, Maria Curie-Skłodowska University, pl. M. Curie-Skłodowskiej 1, 20-031 Lublin, Poland \\ The Monte Carlo method based model of the ionization in the hot cavity taking into account radioactive \\ decay of nuclides is presented. The code upgraded compared to the previous version enables calculation of the ion \\ source ionization efficiency not only for different geometries and temperatures of the ionizer, extraction voltages \\ etc. but also gives opportunity of setting different values of the nuclide half-life and its sticking time, which may \\ be considered as the main factor determining the time that a particle spends in the ion source. The results of \\ calculations are presented together with the discussion of the radioactive decay on the ion source performance.
}

DOI: 10.12693/APhysPolA.123.847

PACS: 07.77.Ka, 07.05.Tp, 34.35. $+\mathrm{a}$

\section{Introduction}

Surface ionization ion sources of various designs are widely used in many fields of science and technology including nuclear spectroscopy, solid state physics. Sources of that kind, very often equipped with a hot cavity ionizer, were invented many years ago [1]. They are, however, still considered as useful equipment, and attract attention of scientists and engineers [2-4], due to their numerous advantages. Surface ionization ion sources are characterized by high purity and low emittance of the obtained ion beam [5]. Also the time the nuclides spend in the ion source is very short, which is of great importance in the case of nuclear spectroscopy of short-lived isotopes. The other advantages that should be mentioned are: relatively high ionization efficiency and, consequently, very small amounts of substance needed for obtaining ion beams of satisfactory intensity as well as their robust construction.

In the paper we focus on surface ionization ion sources with a hot ionization cavity. Theoretical models of ionization inside hot cavity were extensively studied [6-9]. Recently, both theoretical [10], and numerical models of ionizations have been developed [11, 12] based on the conception that high ionization efficiency is an effect of multiple collisions with hot ionizer walls, and also electron impact ionization [13]. The model enabled the studies of ionization efficiency as a function of many factors, ionizer geometry (including the spherical one [14]), extraction system properties, ionizer temperature, ionizer/ sample combination etc. However, the model was adequate mostly for stable or long-lived isotopes, as it did not take into account the effects of radioactive decay of nuclides during their stay in the ion source, which restricted severely its usefulness in the field of nuclear

\footnotetext{
*e-mail: mturek@kft.umcs.lublin.pl
}

spectroscopy. This problem has been solved in the presented upgrade of the model: particles undergo radioactive decay, which is implemented using the Monte Carlo method. The code takes into account not only the particle time of flight but also the time particle stays on the surface of the ionizer. Hence, the code enables the studies of the efficiency changes with the ionizer and extraction system geometry, temperature etc. also for short-lived isotopes.

The brief description of the numerical model is given in the paper. The results of calculation of ionization efficiency as functions of nuclide half-life are presented. The influence of the nuclide sticking time, which appears to be the main factor determining the time a particle stays in the cavity, on ion source performance is under investigation. The discussion of the changes of ionization efficiency with the length of the ionizer in the case of radioactive nuclides is also included.

\section{Hot cavity ion source - construction and principle of operation}

The most important part of a typical hot cavity surface ionization source is a long, semi-opened tube made of refractory metals (those with high-work function are suitable for positive ion generation) known as an ionizer. A variety of low-work function materials including the compounds $\mathrm{LaB}_{6}, \mathrm{GdB}_{6}, \mathrm{Ir}_{5} \mathrm{Ce}$ were tested for effective and selective production of negative ions [15]. The ionizer is usually heated to very high working temperature $T$ $(\approx 2500-3000 \mathrm{~K})$ ohmically or using intense electron ion beams. Atoms introduced into the ionizer collide with hot walls and are ionized with a probability described by the Saha-Langmuir formula [16] - the rate of ions and atoms, known as a ionization degree, could be written as

$$
\alpha=G \exp \left(-\left(V_{\mathrm{i}}-\phi_{\mathrm{e}}\right) / k T\right),
$$

where $V_{\mathrm{i}}$ and $\phi_{\mathrm{e}}$ are the ionization potential of an atom and the work function of the ionizer, respectively, and $G=g_{+}\left(1-r_{+}\right) / g_{\mathrm{a}}\left(1-r_{\mathrm{a}}\right)$, where $r_{\mathrm{a}}$ and $r_{+}$are the reflection coefficients of neutral and charged particles on the 
surface, respectively, $g_{+}$and $g_{\mathrm{a}}$ are the statistical weights of the ground levels of both species related to their total spin numbers. The above formula remains valid also for negative ions, however, $V_{\mathrm{i}}-\phi_{\mathrm{e}}$ has to be substituted by $\phi_{\mathrm{e}}-E_{\mathrm{a}}$, where $E_{\mathrm{a}}$ is the electron affinity of an atom. The frequently used quantity describing ionization is the ionization coefficient $\beta=\alpha /(1+\alpha)-$ the probability of ionization during a single collision. The reader should not confuse the above-defined quantities with the total source ionization efficiency, the quantity defined as the ratio of the number of desired ions and the number of all particles passing through the extraction opening.

Obtaining high total ionization efficiencies in a hot cavity source, much higher than $\beta$ predicted by (1), is possible due to multiple collisions of particles with the ionizing surface. It is also desirable, especially in the case of short-lived isotopes, that ions are extracted from the source as soon as possible after their creation.

\section{Numerical model}

The numerical code is an upgraded version of that previously described [12]. The semi-opened cylindrical ionizer of the length $L$ and the radius $r$ has been considered (see Fig. 1). Ions are attracted by a single flat extraction electrode (voltage $-U_{\text {ext }}$ ) at the distance $d$ from the ionizer opening. The code solves the classical equation of motion of test particles inside the ionizer using the fourth order Runge-Kutta method [17]. The potential distribution is determined by the ionizer and the extraction electrode voltages and it is found by successive over-relaxation method, as in our other models of ion sources [18-20]. The simulation area is covered by numerical grid with the cell sizes $0.1 \times 0.05 \times 0.05 \mathrm{~mm}^{3}$. The potential at sites of test particles is worked out by the linear interpolation of potential values in the nearest grid nodes. The code detects whether the particle touched the ionizer surface or not and the Monte Carlo method based subroutine decides on the particle ionization or neutralization, depending on the ionization parameter.



Fig. 1. Schematic view of the simulated system.

On average the particle stays at the hot surface for a $\tau_{\mathrm{s}}$ - so-called sticking time - this is a new feature of the code. One should remember that the value of $\tau_{\mathrm{s}}$ varies with the surface temperature [21]. It is assumed that atoms are emitted from the hot ionizer surface according to the cosine distribution with the velocities corresponding to the temperature of the ionizer. The particle keeps on moving until another hit or extraction. Multiple collisions with hot walls are basic mechanism leading to high ionization efficiencies in the described model. For simplicity, the effects of electron impact ionization (it may become important for extremely high $k T$ and substances of low $\beta$ ), as well as particle-particle collisions are neglected.

However, the model takes into account the fact that the desired nuclides (both ions and neutrals) undergo radioactive decay with a half-life $\tau_{1 / 2}$. It is assumed that each primary nuclide decays after some time $t_{\mathrm{dec}}$ :

$$
t_{\mathrm{dec}}=\tau_{1 / 2} \ln \mathrm{RND},
$$

where RND is the normal pseudorandom number. Hence, according to the upgraded model, the yield of the desired (primary) ions would be diminished the more the longer particle stays in the ion source. The code registers the numbers of ions of primary and secondary nuclides $\left(N_{\mathrm{p}+}\right.$ and $\left.N_{\mathrm{s}+}\right)$ as well as neutrals $\left(N_{\mathrm{p} 0}\right.$ and $\left.N_{\mathrm{s} 0}\right)$.

\section{Simulation results}

As the first, the changes of the ion source efficiency with the nuclide half-life were examined. A cylindrical ionizer of the length $L=40 \mathrm{~mm}$ and $r=2 \mathrm{~mm}$ was chosen. The extraction voltage was $U_{\text {ext }}=1 \mathrm{kV}$. The initial velocities of particles corresponded to $k T=0.3 \mathrm{eV}$. The ensemble of 50000 test particles was employed. The mass of the test particle was 150 a.m.u. The simplifying assumption has been made that the particle mass does not change during the radioactive decay. The time step $\Delta t=2 \times 10^{-8} \mathrm{~s}$ was chosen. It should be mentioned that on this stage the sticking time $\tau_{\mathrm{s}}$ was set to zero, for the sake of simplicity.

Figure 2a shows the total ionization efficiency as the function of ionization coefficient for different decay values of the half-life. Total ionization efficiency is defined as the ratio of the number of extracted ions of the desired (primary) nuclide $N_{\mathrm{p}+}$ to the number of all extracted particles

$$
\beta_{\mathrm{s}}=\frac{N_{\mathrm{p}+}}{N_{\mathrm{p}+}+N_{\mathrm{s}+}+N_{\mathrm{p} 0}+N_{\mathrm{s} 0}} .
$$

The half-life was changed in the range $0.1 \mathrm{~ms}-1 \mathrm{~s}$. In all cases increase of ionization efficiency with $\beta$ was observed. One can see the influence of the radioactive decay on the source efficiency. For $\tau_{1 / 2}$ greater than $0.1 \mathrm{~s}$ the influence could be neglected. However, for $\tau_{1 / 2}$ in the range of $1 \mathrm{~ms}$ the loss of desired nuclides by radioactive decay reduces the efficiency by approximately $50 \%$. In the case of $\tau_{1 / 2}=0.1 \mathrm{~ms}$ the total efficiency is smaller than the ionization coefficient predicted by (1) and barely reaches 0.1 for large $\beta$. Figure $2 \mathrm{~b}$ presents the ratio of extracted secondary and primary ions $\delta_{\text {rec }}$ as a function of $\beta$. As one can see, this ratio depends very little on $\beta$ for large enough $\tau_{1 / 2}$ (the change is $\approx 10 \%$ for $\tau_{1 / 2}=0.01 \mathrm{~s}$ ). The decrease of $\delta_{\mathrm{dec}}$ with $\beta$ is more 




Fig. 2. Total ionization efficiency as the function of ionization coefficient for different values of $\tau_{1 / 2}$ (a), the ratio of secondary and primary ions as the function of $\beta$ for different values of $\tau_{1 / 2}$ (b), the mean time that a particle stays in the cavity (c) - the case of $\tau_{\mathrm{s}}=0$.

prominent for $\tau_{1 / 2}=0.1 \mathrm{~ms}$ and reaches more than 50 across two decades. This is due to the fact that particles characterized by a high ionization coefficient are ionized and extracted faster than they undergo radioactive decay. This is in a good agreement with the results presented in Fig. 2c. It shows the mean time of flight until the extraction $\langle t\rangle$ as a function of the ionization parameter. The mean time decreases fast with $\beta$ (from $\approx 1.13 \mathrm{~ms}$ for $\beta=0.01$ to $\approx 0.75$ for $\beta=1$. The impact of radioactive decay on the ionization efficiency, visible in Fig. 2 as lowering of the $\beta_{\mathrm{s}}(\beta)$ curves, becomes important when $\tau_{1 / 2}$ is comparable to the mean flight time (of the order $1 \mathrm{~ms}$ in the considered case).

The results shown in Fig. 2 suggest that a proper choice of ionizer material and its temperature could be of major importance for obtaining high yields of short-lived isotope ions.

In the next stage, the influence of sticking time on the ionizer surface was investigated. The simplifying assumption has been made that every time a particle touches the hot wall, it remains for $\tau_{\mathrm{s}}$ on its surface. The value of $\tau_{\mathrm{s}}$ varied in a broad range (up to $10 \mathrm{~ms}$ ) as it strongly depends on the ionizer temperature and the ionizer/ionized atom combination, which is described by the Frenkel equation [21]. The other simulation parameters were unchanged, compared to the previous case.

Figure 3a shows the changes of ionization efficiency due to the length of sticking time. Calculations were done for $\tau_{1 / 2}=0.01 \mathrm{~s}$. As the particle undergoes many (several tens or hundreds) collisions with the ionizer, the lowering of $\beta_{\mathrm{s}}(\beta)$ curve is visible even for $\tau_{\mathrm{s}}=1 \mu \mathrm{s}$. Setting $\tau_{\mathrm{s}}=10 \mu \mathrm{s}$ leads to the ionization efficiencies reduced by approximately 50, whilst the sticking times in the millisecond range reduce the source efficiency by an order of magnitude (compared to the idealized $\tau_{\mathrm{s}}=0$ case).



Fig. 3. Total ionization efficiency as the function of ionization coefficient for different values of $\tau_{\mathrm{s}}$ for $\tau_{1 / 2}=$ $0.01 \mathrm{~s}(\mathrm{a})$, the ratio of secondary and primary ions as the function of $\beta$ for different values of $\tau_{\mathrm{s}}(\mathrm{b})$, the mean time a particle stays on the ionizer surface, the dashed line represents the mean flight time (c).

Also the ratio $\delta_{\text {rec }}$ reaches very high values for long sticking times ( 1 ion of the desired kind per 100 of extracted particles). Fortunately, this could be improved by either increasing the temperature of the ionizer or a more adequate choice of the ionizer material/coating. Reducing the sticking time by the order of magnitude may lead to the decrease of $\delta_{\text {rec }}$ ratio by 10 times for large $\beta$, or even by the factor of 100 for smaller $\beta$ (see Fig. 3b). Even for relatively short sticking time $(100 \mu \mathrm{s})$ the contribution of the mean time $\left\langle t_{\mathrm{s}}\right\rangle$ spent on the surface of ionizer could be $\approx 10$ times larger than the time of its flight, as shown in Fig. 3c. As in the previous case one can observe slight decrease of $\left\langle t_{\mathrm{s}}\right\rangle$ - the substances of larger $\beta$ are ionized and extracted faster, consequently, the number of collisions with the ionizer walls is on the average smaller than in the case of hard-to-ionize elements.
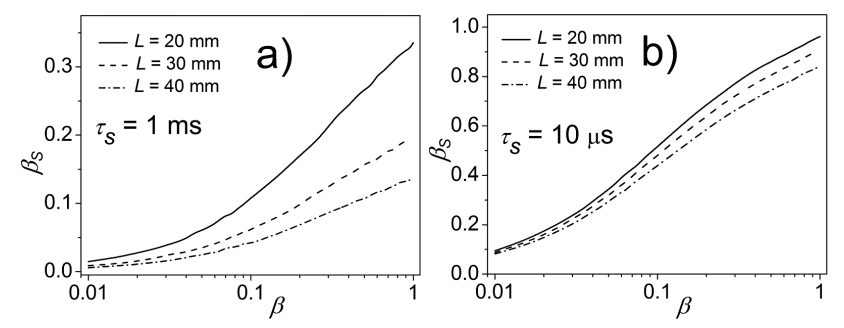

Fig. 4. Total ionization efficiency as the function of ionization coefficient for different $L$ : the cases of $\tau_{\mathrm{s}}=$ $1 \mathrm{~ms}(\mathrm{a})$ and $\tau_{\mathrm{s}}=10 \mu \mathrm{s}(\mathrm{b})$.

Influence of the ionizer length $L$ on the ionization efficiency was also under investigation. The results for the sticking time $\tau_{\mathrm{s}}=1 \mathrm{~ms}$ are shown in Fig. 4a. The half-life time was set to $\tau_{1 / 2}=0.01 \mathrm{~s}$, as in the previous 
case. One can see that the ionization efficiency is higher for shorter ionizers - it increases more than twice when $L$ is decreased from $40 \mathrm{~mm}$ to $20 \mathrm{~mm}$. This is due to the shorter time that the particle needs to get the extraction opening in the case of shorter ionizer. This effect may be important in the case of radioactive nuclides and relatively long sticking times. In the case of short $\tau_{\mathrm{s}}$ (or relatively long $\tau_{1 / 2}$ ) the influence of $L$ is much smaller (see Fig. 4b). One should keep in mind that in the case of non-radioactive nuclides one observes also a slight increase of ion source efficiency, mostly due to the smaller volume where the particles roam accidentally, until they are caught by the extraction field. One may expect that profits from shorter ionizer are the greater the shorter is $\tau_{1 / 2}$ and the longer time a particle stays on the ionizer surface.

Changes of ionization efficiency with the extraction voltage were also studied. It was found that in the case of stable isotopes the extraction voltage in the range of several thousands of volts is sufficient, while in the case of short-lived isotopes higher voltages are required to achieve saturation of ion current.

\section{Conclusions}

The Monte Carlo numerical model of ionization in a hot cavity ion source has been upgraded in order to take into account the losses caused by radioactive decay. The code takes into account not only the particle time of flight, but also the time it stays on the ionizer surface. It was found that the reduction of the sticking time (e.g. by the proper choice of the ionizer coating and temperature) may significantly increase ion yields in the case of short-lived isotopes. The simulation results show that shorter ionizers are much more effective for short lived nuclides. The model could be considered as a useful tool supporting the studies of processes inside the surface ion sources, as well as production of beams of radioactive nuclides. The further development of the code is planned, including modelling of the diffusion of nuclides out of the irradiated target, as well as the modifications enabling the description of the target built of a large number of thin foils.

\section{Acknowledgments}

This work was supported by the Polish Ministry of Science and Higher Education, grant No. N N515 246637.

\section{References}

[1] G.J. Beyer, E. Herrmann, A. Piotrowski, V.I. Raiko, H. Tyroff, Nucl. Instrum. Meth. 96, 437 (1971).

[2] V.N. Panteleev, Rev. Sci. Instrum. 75, 1602 (2004).

[3] G.D. Alton, Y. Liu, D.W. Stracener, Rev. Sci. Instrum. 77, 03A 711 (2006).

[4] U. Köster, O. Arndt, E. Bouquerel, V.N. Fedoseyev, H. Franberg, A. Joinet, C. Jost, I.S.K. Kerkines, R. Kirchner, The TARGISOL Collaboration, Nucl. Instrum. Methods Phys. Res. B 266, 4229 (2008).

[5] G.D. Alton, Y. Liu, H. Zaim, S.N. Murray, Nucl. Instrum. Meth. Phys. Res. B 211, 425 (2003).

[6] A. Latuszyński, V.I. Raiko, Nucl. Instrum. Meth. Phys. Res. 125, 61 (1975).

[7] R. Kirchner, Nucl. Instrum. Meth. Phys. Res. 186, 275 (1981).

[8] M. Huyse, Nucl. Instrum. Meth. Phys. Res. 215, 1 (1983).

[9] R. Kirchner, Nucl. Instrum. Meth. Phys. Res. A 292, 203 (1990).

[10] A. Latuszyński, K. Pyszniak, A. Droździel, M. Turek, D. Mączka, J. Meldizon, Vacuum 81, 1150 (2007).

[11] M. Turek, K. Pyszniak, A. Drozdziel, J. Sielanko, Vacuum 82, 1103 (2008).

[12] M. Turek, A. Droździel, K. Pyszniak, D. Mączka, B. Słowiński, Rev. Sci. Instrum. 83, 023303 (2012).

[13] M. Turek, K. Pyszniak, A. Droździel, Vacuum 83, S260 (2009).

[14] M. Turek, Acta Phys. Pol. A 120, 188 (2011).

[15] M. Menna, R. Catherall, J. Lettry, E. Noah, T. Stora, and the ISOLDE collaboration, Nucl. Instrum. Meth. Phys. Res. B 266, 4391 (2008).

[16] The Physics and Technology of Ion Sources, Ed. I.G. Brown, Wiley-VCH, Berlin 2004.

[17] W.H. Press, B.P. Flannery, S.A. Teukolsky, W.T. Vetterling, Numerical Recipes: The Art of Scientific Computing, Cambridge University Press, Cambridge 1986.

[18] A. Pyszniak, A. Droździel, M. Turek, A. Latuszyński, D. Maczka, J. Sielanko, Yu.A. Vaganov, Yu.V. Yushkevich, Instrum. Exp. Techn. 50, 552 (2007).

[19] M. Turek, K. Pyszniak, A. Drozdziel, J. Sielanko, A. Latuszynski, D. Maczka, Yu.A. Vaganov, Yu.V. Yushkevich, Instrum. Exp. Techn. 52, 90 (2009).

[20] M. Turek, S. Prucnal, A. Drozdziel, K. Pyszniak, Rev. Sci. Instrum. 80, 043304 (2009).

[21] A. Zangwill, Physics at Surfaces, Cambridge University Press, Cambridge 1988. 